\title{
Analysis of Linear Viscoelastic Behavior of Microphase-Separated ABC Three-Block Polymer Systems by Finite-Element Method
}

\author{
Hiroshi WatAnABE and Tadao KotaKA* \\ Department of Macromolecular Science, College of Science, Osaka University, \\ Toyonaka, Osaka 560, Japan.
}

(Received July 14, 1980)

\begin{abstract}
A finite element method was extended and applied to a microphase-separated poly(styrene- $b$-butadiene- $b$-4-vinylpyridine) (SBP) three-block polymer for analysis of temperature dispersion of dynamic viscoelastic response under a small-amplitude sinusoidal oscillation. The application of this method to a multiphase viscoelastic system requires a knowledge of the typical relaxation spectrum of an amorphous polymer and the temperatures of the onset of glass transitions of the constituent homopolymers as well as the morphology of the system. Two typical morphologies were examined. One was a "ball-in-a-box" structure in which a poly(4-vinylpyridine) (P) ball sits in a polystyrene (S) box stuffed the gap with polybutadiene (B) blocks, and the other a "three-layer-lamellar" morphology in which three phases alternate in the manner ... SBP. PBS. SBP .... In the temperature dispersion of the moduli, the former shows only two transitions due to the glass transitions of $B$ and $S$ phases, while the latter shows three transitions due to those of all three phases. The finite-element-method analysis discribes well or at least semiquantitatively the features of such three phase systems having particular morphologies.

KEY WORDS Matrix Finite Element Method / Linear Viscoelasticity / ABC Three-Block Polymer / Poly(styrene- $b$-butadiene- $b$-4-vinylpyridine) / Three Phase Domain Morphology /
\end{abstract}

Because of the recent sophistication taken on by high-performance electronic computers, the finiteelement method ${ }^{1,2}$ is becoming increasingly a more powerful tool in continuum mechanics. The finiteelement method has been applied to stress-strain analysis within a body of complex shape made from glasses and metals. The method has also been applied to the large elastic-deformation behavior of vulcanized rubbers. ${ }^{3,4}$ Here we would like to apply this method to analyses of linear viscoelastic responses of microphase-separated polymeric alloys having regular domain morphologies. The systems we are specifically interested in are of a poly(styrene$b$-butadiene- $b$-4-vinylpyridine) (SBP) three-block polymer that has with nearly a $1: 1: 1.5$ composition. ${ }^{5,6}$ An SBP film cast from $\mathrm{CHCl}_{3}$ exhibits a unique "ball-in-a-box" morphology, in which a poly(4-vinylpyridine) ball sits in a polystyrene box stuffed with soft polybutadiene blocks, ${ }^{5}$ while an

* To whom correspondence should be addressed.
SBP film cast from butyraldehyde (BA) $/ \mathrm{CHCl}_{3}$ (9:1) mixture exhibits a "three-layer lamellar" morphology in which three phases alternate in the manner $\cdot$ SBP.PBS $\cdot$ SBP $\cdot{ }^{6}$ Figure 1 shows the morphologies of such SBP films.

When the finite-element method is applied to a viscoelastic body instead of an elastic body, it has to be modified so that the past memory can be incorporated so as to describe present stress level. First, we shall discuss this modification of the finiteelement method. Then we apply the modified method to the analysis of the viscoelastic properties of SBP films under a sinusoidal shear deformation with a small amplitude.

\section{APPLICATION OF METHOD TO A LINEAR VISCOELASTIC BODY}

\section{Constitutive Equation}

Before constructing a concrete formulae for a finite-element method analysis, a knowledge of the 


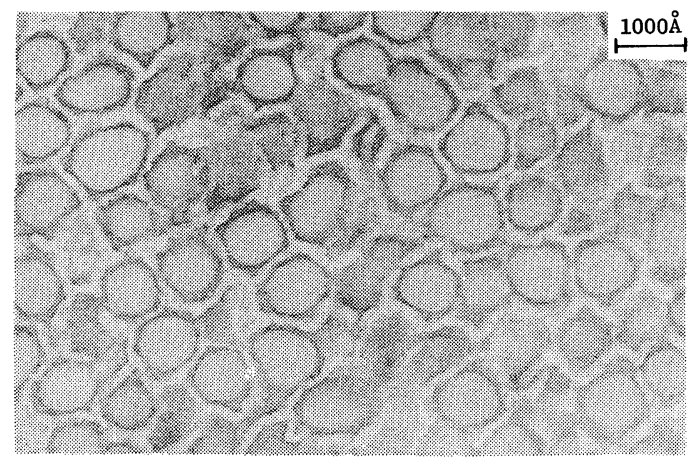

SBP cast from $\mathrm{CHCl}_{3}$

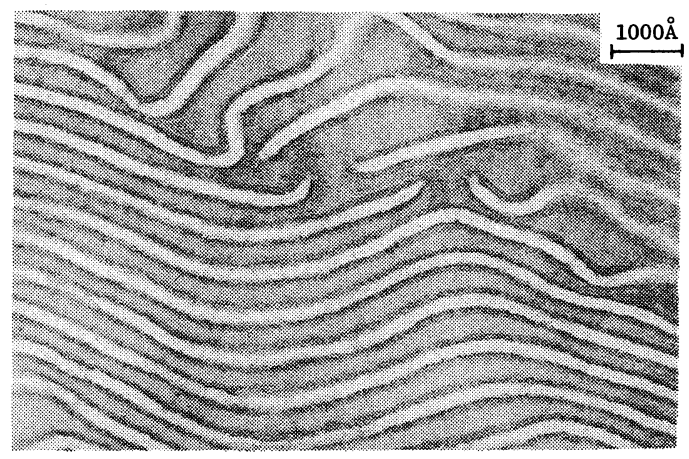

SBP cast from $\mathrm{BA} / \mathrm{CHCl}_{3}$

Figure 1. Electron micrographs of SBP-1 specimens cast from $\mathrm{CHCl}_{3}$ and butylaldehyde (BA) $/ \mathrm{CHCl}_{3}(9: 1$ $\mathrm{v} / \mathrm{v})$ mixture.

constitutive equation of the material to be treated is necessary. For a linear viscoelastic body, the stress tensor $\boldsymbol{\sigma}(t)$ is represented as a convolution of the relative strain tensor $\lambda\left(t ; t^{\prime}\right){ }^{7}$

$$
\boldsymbol{\sigma}(t)=\int_{-\infty}^{t} \mu^{\prime}\left(t-t^{\prime}\right)\left[\lambda\left(t ; t^{\prime}\right)-\boldsymbol{E}\right] \mathrm{d} t^{\prime}
$$

where $\boldsymbol{E}$ is a unit tensor. The response function $\mu^{\prime}$ and the relative strain tensor $\lambda\left(t ; t^{\prime}\right)$ are related to the relaxation function $\phi$ and the displacement tensor $\gamma(t)$ as follows.

$$
\begin{aligned}
\mu^{\prime}(t) & =-\frac{\mathrm{d} \phi(t)}{\mathrm{d} t} \\
\lambda\left(t ; t^{\prime}\right) & =\gamma(t) \gamma\left(t^{\prime}\right)^{-1} \gamma\left(t^{\prime}\right)^{+-1} \gamma(t)^{+}
\end{aligned}
$$

For a small deformation eq 3 is reduced to eq 4 :

$$
\lambda\left(t ; t^{\prime}\right)=\left[\boldsymbol{a}(t)+\boldsymbol{a}(t)^{+}\right]-\left[\boldsymbol{a}\left(t^{\prime}\right)+\boldsymbol{a}\left(t^{\prime}\right)^{+}\right]+\boldsymbol{E}
$$

The tensor $\boldsymbol{a}(t)=\gamma(t)-\boldsymbol{E}$ is a small displacement tensor.

For a sinusoidal shear deformation of a continuum (single-phase) viscoelastic body, the stress and strain tensors can be treated as two dimensional quantities with a single response function $\mu^{\prime}\left(t-t^{\prime}\right)$, as long as the amplitude of deformation remains small. For the sake of convenience, the stress tensor $\boldsymbol{\sigma}(t)$ and the strain tensor $\varepsilon(t)$ are represented as eq 5 and 6

$$
\begin{gathered}
\boldsymbol{\sigma}(t)=\left|\begin{array}{l}
\sigma_{x x}(t) \\
\sigma_{y y}(t) \\
\sigma_{x y}(t)
\end{array}\right| \\
\varepsilon(t)=\left|\begin{array}{l}
\varepsilon_{x x}(t) \\
\varepsilon_{y y}(t) \\
\varepsilon_{x y}(t)
\end{array}\right|
\end{gathered}
$$

with $\varepsilon_{x x}$ etc. the components of the small strain tensor $a(t)+a(t)^{+}$. The relative strain tensor $\lambda\left(t ; t^{\prime}\right)$ is then represented by the components of $\varepsilon(t)$ as follows.

$$
\lambda\left(t ; t^{\prime}\right)=\left|\begin{array}{l}
2 \varepsilon_{x x}(t)-2 \varepsilon_{x x}\left(t^{\prime}\right)+1 \\
2 \varepsilon_{y y}(t)-2 \varepsilon_{y y}\left(t^{\prime}\right)+1 \\
\varepsilon_{x y}(t)-\varepsilon_{x y}\left(t^{\prime}\right)
\end{array}\right|
$$

The constitutive equation (1) is now rewritten as

$$
\boldsymbol{\sigma}(t)=\int_{0}^{\infty} \mu^{\prime}\left(t^{\prime}\right) \mathrm{d} t^{\prime} \boldsymbol{Q} \varepsilon(t)-\boldsymbol{Q} \int_{-\infty}^{t} \mu^{\prime}\left(t-t^{\prime}\right) \boldsymbol{\varepsilon}\left(t^{\prime}\right) \mathrm{d} t^{\prime}
$$

where $Q$ is a matrix given by

$$
\boldsymbol{Q}=\left|\begin{array}{lll}
2 & 0 & 0 \\
0 & 2 & 0 \\
0 & 0 & 1
\end{array}\right|
$$

\section{Mechanical Characteristics of an Element ${ }^{1,2}$}

When the finite-element method is used to analyze the mechanical behavior of a body, the body is replaced by a "total structure" consisting of a finite number of elements with definite shape. The mechanical behavior of the body is then analyzed in relation to the displacements of nodes representing the elements and forces acting thereon. Triangular elements are suitable for treating two-dimensional problems. For example, a "toral structure" is divided into triangles to yield $N$ nodes, such as shown in Figure 2. The nodes are numbered from one to $N$, and three nodes $i, j, k(1 \leqq i<j<k \leqq N)$ belong to the $m$-th element. If a uniform linear deformation is assumed within each element, the strain $\varepsilon_{\mathrm{m}}$ in this element is related to the displace- 


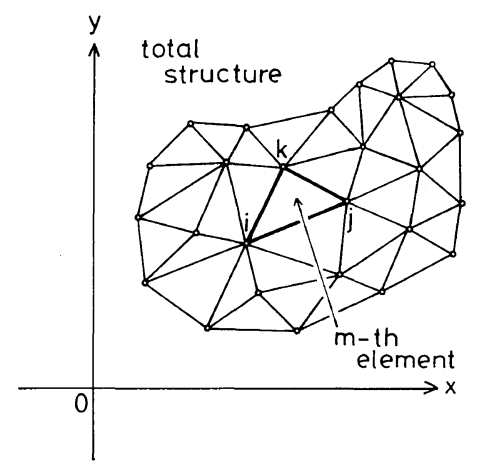

Figure 2. Schmematic diagram of a "total structure" divided into triangular elements.

ments of the three nodes $\delta_{\mathrm{m}}(t)=\left(u_{i}, v_{i}, u_{j}, v_{j}, u_{k}, v_{k}\right)^{+}$,

$$
\varepsilon_{\mathrm{m}}(t)=\frac{1}{2 \Delta_{\mathrm{m}}}\left|\begin{array}{llllll}
b_{i} & 0 & b_{j} & 0 & b_{k} & 0 \\
0 & c_{i} & 0 & c_{j} & 0 & c_{k} \\
c_{i} & b_{i} & c_{j} & b_{j} & c_{k} & b_{k}
\end{array}\right|\left|\begin{array}{l}
u_{i}(t) \\
v_{i}(t) \\
u_{j}(t) \\
v_{j}(t) \\
u_{k}(t) \\
v_{k}(t)
\end{array}\right|
$$

$$
=B_{\mathrm{m}} \boldsymbol{\delta}_{\mathrm{m}}(t)
$$

where $\Delta_{\mathrm{m}}$ is the area of the $m$ th element, $u_{p}(t)$ and $v_{p}(t)$ are displacements of the $p$ th node at time $t$ in the $x$ - and $y$-directions, respectively, and $b_{p}$ and $c_{p}$ are related to the coordinates of nodes $\left(x_{p}, y_{p}\right)^{+}$ $(p=i, j, k)$ as follows.

$$
\begin{array}{ll}
b_{i}=y_{j}-y_{k} & c_{i}=x_{k}-x_{j} \\
b_{j}=y_{k}-y_{i} & c_{j}=x_{i}-x_{k} \\
b_{k}=y_{i}-y_{j} & c_{k}=x_{j}-x_{i}
\end{array}
$$

The above assumption is reasonable when the deformation is small and the material in this element is uniform and isotropic. From eq 8 and $10, \sigma_{\mathrm{m}}$ is related to $\boldsymbol{\delta}_{\mathrm{m}}$ as

$$
\begin{aligned}
& \boldsymbol{\sigma}_{\mathrm{m}}(t)=\int_{0}^{\infty} \mu_{\mathrm{m}}^{\prime}\left(t^{\prime}\right) \mathrm{d} t^{\prime} \boldsymbol{Q} \boldsymbol{B}_{\mathrm{m}} \boldsymbol{\delta}_{\mathrm{m}}(t) \\
&-\boldsymbol{Q} \boldsymbol{B}_{\mathrm{m}} \int_{-\infty}^{t} \mu_{\mathrm{m}}^{\prime}\left(t-t^{\prime}\right) \boldsymbol{\delta}_{\mathrm{m}}\left(t^{\prime}\right) \mathrm{d} t^{\prime}
\end{aligned}
$$

Here $\mu_{\mathrm{m}}^{\prime}$ denotes the response function of the material in the $m$ th element.

In order to obtain the relation between the force $\boldsymbol{F}_{\mathrm{m}}(t)$ acting on the $m$ th element and the displacements of the three nodes $\delta_{\mathrm{m}}(t)$, the principle of virtual displacement is used. A virtual work $\delta W^{*}$ caused by a virtual displacement $\delta_{\mathrm{m}}^{*}$ and a virtual strain $\boldsymbol{\varepsilon}_{\mathrm{m}}^{*}=\boldsymbol{B}_{\mathrm{m}} \boldsymbol{\delta}_{\mathrm{m}}^{*}$ is given as

$$
\begin{aligned}
\delta W^{*} & =\delta_{\mathrm{m}}^{*+} \boldsymbol{F}_{\mathrm{m}}(t) \\
& =h \iint \varepsilon_{\mathrm{m}}^{*+} \boldsymbol{\sigma}_{\mathrm{m}}(t) \mathrm{d} x \mathrm{~d} y
\end{aligned}
$$

Here, $h$ is the thickness of the element, and the integration is carried out over the surface of this element. From eq 12 and 13, mechanical characteristics of the $m$ th element is obtained as the relation between $\boldsymbol{\delta}_{\mathrm{m}}$ and $\boldsymbol{F}_{\mathrm{m}}$ as follows.

$$
\boldsymbol{F}_{\mathrm{m}}(t)=h \Delta_{\mathrm{m}} \boldsymbol{B}^{+}{ }_{\mathrm{m}} \boldsymbol{Q B} \boldsymbol{B}_{\mathrm{m}}
$$

$$
\begin{aligned}
& \times\left[\int_{\delta t}^{\infty} \mu_{\mathrm{m}}^{\prime}\left(t^{\prime}\right) \mathrm{d} t^{\prime} \boldsymbol{\delta}_{\mathrm{m}}(t)-\int_{-\infty}^{t-\delta t} \mu_{\mathrm{m}}^{\prime}\left(t-t^{\prime}\right) \boldsymbol{\delta}_{\mathrm{m}}\left(t^{\prime}\right) \mathrm{d} t^{\prime}\right] \\
& =\boldsymbol{K}_{\mathrm{m}} \boldsymbol{\delta}_{\mathrm{m}}(t)-\boldsymbol{R}_{\mathrm{m}}(t)
\end{aligned}
$$

In this eq $14 \delta t$ denotes a small time interval. Only the past displacements $\delta_{\mathrm{m}}\left(t^{\prime}\right)\left(t^{\prime}<t\right)$ contribute to $\boldsymbol{R}_{\mathrm{m}}(t)$ if the range of integral is limited as in eq 14 .

\section{Mechanical Characteristics of the "Total Structure"}

In eq 14 the $m$ th element is represented by the three nodes, and therefore $\boldsymbol{F}_{\mathrm{m}}(t), \boldsymbol{\delta}_{\mathrm{m}}(t), \boldsymbol{R}_{\mathrm{m}}(t)$, and $\boldsymbol{K}_{\mathrm{m}}$ are six-dimensional representations. After rewriting the six-dimensional matrices for the $N$ nodes, which are given by $2 \mathrm{~N}$-dimensional representations, eq 14 is summed up for all the elements to give the relation between the force $\boldsymbol{F}$ and the displacement $\boldsymbol{\delta}$ of all the $N$ nodes,

$$
\boldsymbol{F}(t)=\boldsymbol{K} \boldsymbol{\delta}(t)-R(\mathrm{t})
$$

When the displacement of the boundary of the "total structure," i.e., the boundary condition is given, the force $\boldsymbol{F}(t)$ is obtained by solving eq 15. To do this we first classify the $N$ nodes into two classes $\mathrm{A}$ and $\mathrm{B}$, which include, respectively, those within the "total structure" and those on the boundary. We rearrange the components in the tensors in eq 15 so that the components of class A appear in the upper rows of the columns, while the components of class $B$ in the lower half of the columns:

$$
\left|\begin{array}{l}
\boldsymbol{F}^{\mathrm{A}}(t) \\
\boldsymbol{F}^{\mathrm{B}}(t)
\end{array}\right|=\left|\begin{array}{l}
\boldsymbol{K}^{\mathrm{AA}} \boldsymbol{K}^{\mathrm{AB}} \\
\boldsymbol{K}^{\mathrm{BA}} \boldsymbol{K}^{\mathrm{BB}}
\end{array}\right|\left|\begin{array}{l}
\boldsymbol{\delta}^{\mathrm{A}}(t) \\
\boldsymbol{\delta}^{\mathrm{B}}(t)
\end{array}\right|-\left|\begin{array}{l}
\boldsymbol{R}^{\mathrm{A}}(t) \\
\boldsymbol{R}^{\mathrm{B}}(t)
\end{array}\right|
$$

Further, we assume that forces acting on an A-class node are balanced during the deformation, so that 
$F^{\mathrm{A}}(t)$ equals zero. The upper row of eq 16 is then rewritten as follows:

$$
\begin{aligned}
\boldsymbol{\delta}^{\mathrm{A}}(t) & =-\left(\boldsymbol{K}^{\mathrm{AA}}\right)^{-1} \boldsymbol{K}^{\mathrm{AB}} \boldsymbol{\delta}^{\mathrm{B}}(t)+\left(\boldsymbol{K}^{\mathrm{AA}}\right)^{-1} \boldsymbol{R}^{\mathrm{A}}(t) \\
& =-\boldsymbol{Z} \boldsymbol{\delta}^{\mathrm{B}}(t)+\boldsymbol{C}(t)
\end{aligned}
$$

From eq 16 and $17, \boldsymbol{F}^{\mathbf{B}}(t)$ is related only to the displacements of the B-class nodes as

$$
\begin{aligned}
\boldsymbol{F}^{\mathrm{B}}(t) & =\left[\boldsymbol{K}^{\mathrm{BB}}-\boldsymbol{K}^{\mathrm{BA}} Z\right] \boldsymbol{\delta}^{\mathrm{B}}(t)+\left[\boldsymbol{K}^{\mathrm{BA}} \boldsymbol{C}(t)-\boldsymbol{R}^{\mathrm{B}}(t)\right] \\
& =\boldsymbol{A} \boldsymbol{\delta}^{\mathrm{B}}(t)+\boldsymbol{P}(t)
\end{aligned}
$$

Once there is a displacement of the boundary $\delta^{\mathbf{B}}$, i.e., the boundary condition is determined, eq 17 and 18 are solved to give $\boldsymbol{\delta}^{\mathbf{A}}(t)$ and $\boldsymbol{F}^{\mathbf{B}}(t)$. If an elastic body such as that of metals is to be analyzed, $C(t)$ in eq 17 and $\boldsymbol{P}(t)$ in eq 18 , both representing the contribution of the stresses of the past deformation history, are equal to zero, and it is not necessary to obtain $\delta^{\mathrm{A}}(t)$. If a viscoelastic body is to be analyzed, however, $\boldsymbol{\delta}^{\mathbf{A}}\left(t^{\prime}\right)$ and $\boldsymbol{\delta}^{\mathbf{B}}\left(t^{\prime}\right)\left(t^{\prime}<t\right)$ must be obtained to calculate $R_{\mathrm{m}}(t)$ in eq 14 .

\section{COMPARISON OF CALCULATIONS WITH EXPERIMENTS}

\section{Models and Calculations}

The reduction of a three-dimensional problem to a two-dimensional one, as was done just above, may be justified in dealing with the small-amplitude sinusoidal shearing deformation of a continuum single-phase body. However, for the multiphase system which we are examinig, this reduction is highly problematic. We were able to note down, instead of the $2 \mathrm{~N}$-dimensional representation of eq 16, a more general formula involving two material functions corresponding to the shear and bulk moduli or the Young's modulus and the Poisson's ratio for an isotropic elastic body. However, in such a formulation class A and class B components in the deformation tensor could not be adequately resolved. Presumably the volume change of an element due to deformation must be determined by an additional boundary condition such as the balance between the internal and external hydrostatic pressures. The extension of such a treatment to a viscoelastic multiphase system certainly creates further complication, which at the moment we do not know how to resolve, and also there is the difficulty arising from the shortage in the computer capacity.

For two-phase systems, which in most cases consist of hard inclusion phases and soft matrix phases, several theories have been developed for the dynamic mechanical properties ${ }^{8,9}$ on the bases of equivalent mechanical models and of the averaging procedure of Bruggemann ${ }^{10}$ and Kerner. ${ }^{11}$ In some of these theories, two phases were assumed to be incompressible, while in others, the viscoelastic

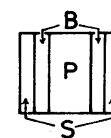

three-layer-lameilar
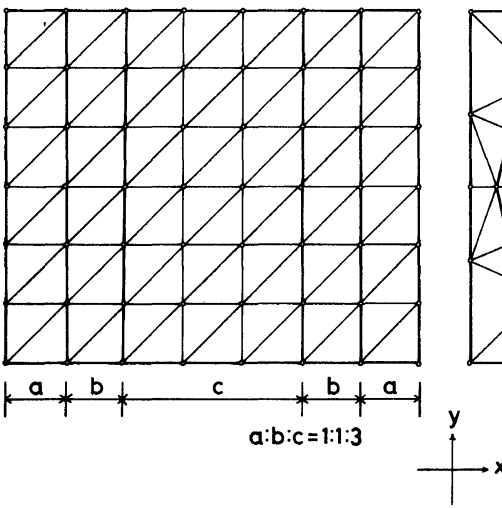

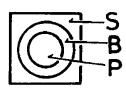

ball-in-a-box

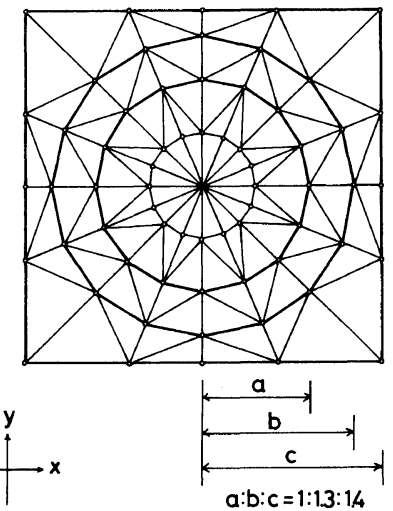

Figure 3. Schematic diagram of "total structures" representing two typical "ball-in-a-box" and "threelayer-lamellar" morphologies of SBP specimens. 
Poisson's ratios were assumed as real constants. Particularly, the averaging-process theories are based on the following two assumptions: the two phases assume a regular morphology which is mostly a viscoelastic sphere in another viscoelastic matrix, and the two-phase system is considered to have the macroscopic mechanical properties of a homogeneous isotropic material, which approach those of the matrix phase as the distance from the dispersed sphere becomes large. ${ }^{8,9}$ Such an analysis certainly remedies any complications arising from, say, the difference between the Poisson's ratios of the two phases, However, the averaging procedure is not applicable to our SBP three-phase systems. Therefore, we have arbitrarily assumed that (i) the effects of volume change in any elements during the sinusoidal shear-deformation of the total structure may be neglected, since the volume change itself must be small as long as the amplitude of oscillation remains small, and that (ii) each element in each phase may be replaced by a uniform, isotropic entity characterized by a single response function similar to that of the corresponding homopolymer. Thus the two typical "ball-in-a-box" and "three-layerlamellar" morphologies of a poly(styrene- $b$ butadiene- $b$-4-vinylpyridine) three-block polymer $(\mathrm{SBP})^{5,6}$ were represented by two models of simple geometry such as shown in Figure 3. Molecular weights $M_{n}$ of each block of the SBP polymer were 20200 (S), 22000 (B), and 32300 (P). ${ }^{5,6}$ The period of oscillating displacement was equally divided into 36 intervals, and eq 17 and 18 were solved numerically so that the forces were balanced at each node. For the calculation of $\boldsymbol{R}_{\mathrm{m}}(t)$ in eq 14, $\delta t$ was put equal to the interval that was $1 / 36$ of a period. The range of numerical integration was limited only to the past three periods, because of the limitation of the computer capacity. When the oscillating stresses reached a stationary level usually achieved after several cycles, the data were taken out and analyzed to evaluate $G^{\prime}$ and $G^{\prime \prime}$.

\section{Response Functions}

The master curves of the dynamic modulus $G^{\prime}(\omega)$ of monodisperse polystyrenes (PS) reduced to $160^{\circ} \mathrm{C}$ are shown in Figure 4. Solid lines in the lower and higher frequency ranges in Figure 4 are the data reported by Onogi et al. ${ }^{12}$ and Soen et al., ${ }^{13}$ respectively. The broken lines in the transition region were obtained by interpolation between the

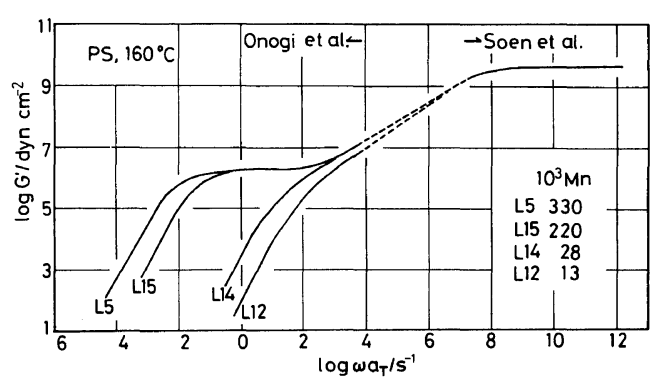

Figure 4. Master curves of dynamic modulus $G^{\prime}$ of polystyrenes reduced to $160^{\circ} \mathrm{C}$.

two solid lines. The reported values of $10^{3} M_{n}$ of L5, L15, L14, and L12 by Onogi et al. were 330, 220, 28, and 13, respectively. The data by Soen et al. obtained for a sample with $M_{n}=180000$ at $100^{\circ} \mathrm{C}$ were reduced to $160^{\circ} \mathrm{C}$ by the WLF relation: ${ }^{14}$

$$
\log a_{\mathrm{T}}=-\frac{17.44\left(T-T_{\mathrm{g}}\right)}{51.6+T-T_{\mathrm{g}}}
$$

When the frequency is high or the temperature is low, the dynamic behavior of PS is assumed to be independent of molecular weight.

From the master curves shown in Figure 4, the response functions $\mu^{\prime}$ and the relaxation spectra $H$ are obtained by the first-order approximation, respectively, as

$$
\begin{aligned}
& \mu^{\prime}(t)=\frac{1}{t}\left[\frac{\mathrm{d} G^{\prime}(\omega)}{\mathrm{d} \ln \omega}\right]_{\omega=1 / t} \\
& H(\tau)=\left[\frac{\mathrm{d} G^{\prime}(\omega)}{\mathrm{d} \ln \omega}\right]_{\omega=1 / \tau}=\left.t \mu^{\prime}(t)\right|_{t=\tau}
\end{aligned}
$$

The results are shown in Figure 5. The response functions are approximately a single-relaxationtime type in a range $t<10^{-9} \mathrm{~s}$, and assumed to be zero in a range $t>10^{4} \mathrm{~s}$ at $160^{\circ} \mathrm{C}$. In the latter calculations, the temperature dependence of $\mu^{\prime}$ is assumed as follows. The shift factor $a_{\mathrm{T}}$ is assumed as in eq 19 when $T>T_{\mathrm{g}}$, and to be unity when $T \leqq T_{\mathrm{g}}$.

$$
\left.\mu^{\prime}(t)\right|_{T}=\left.\frac{1}{a_{\mathrm{T}}} \mu^{\prime}\left(t / a_{\mathrm{T}}\right)\right|_{T_{\mathrm{g}}}
$$

\section{Frequency-Dispersion Behavior of PS}

In order to determine the accuracy of the calculation, the frequency-dispersion behavior of PS at $160^{\circ} \mathrm{C}$ was calculated and compared with the measured results by Onogi et al. ${ }^{12}$ For L5 and L15 


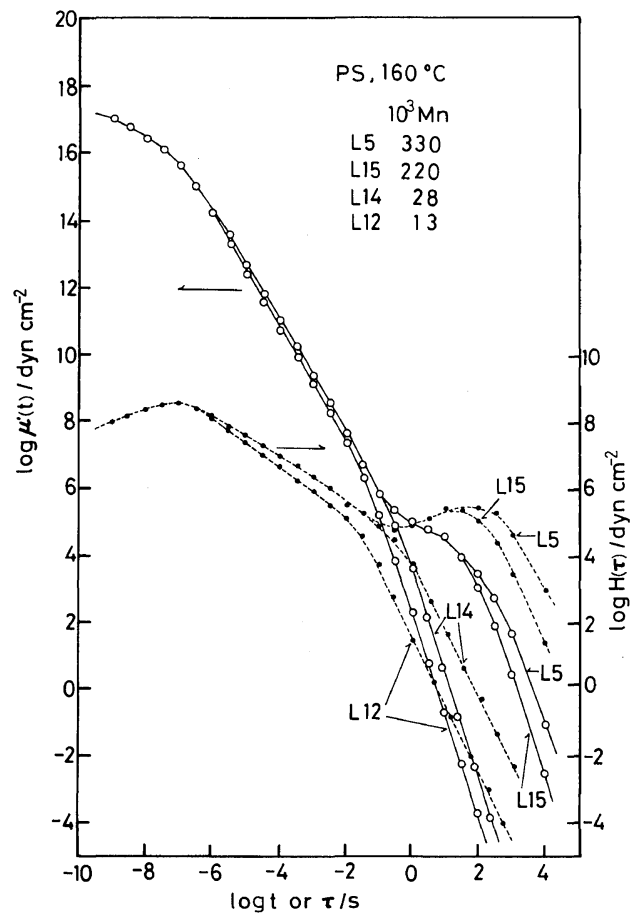

Figure 5. Response functions and relaxation spectra of polystyrenes $\mathrm{L} 5, \mathrm{~L} 15, \mathrm{~L} 14$, and $\mathrm{L} 12$, reduced to $160^{\circ} \mathrm{C}$. These curves were calculated from the $G^{\prime}$ master curves shown in Figure 4.

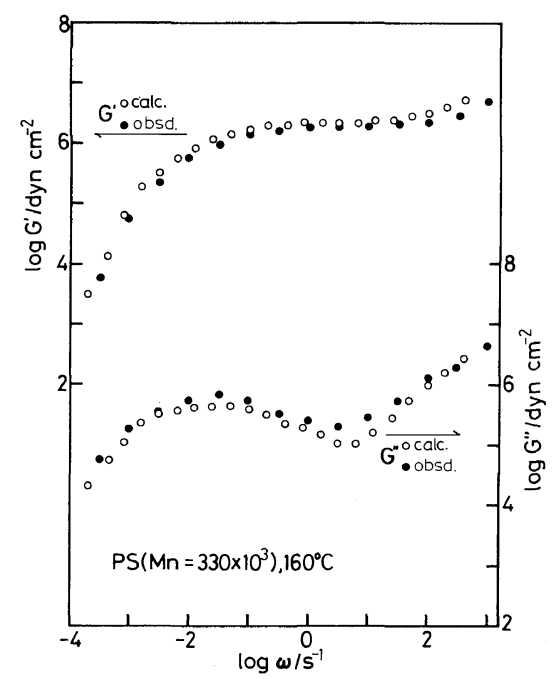

Figure 6. Comparison between calculated and observed $^{8}$ frequency-dispersion behavior of a polystyrene $\left(M_{n}=330000\right)$ at $160^{\circ} \mathrm{C}$. the calculations were done without difficulty. For L14 and L12, however, there were large numerical errors involved: $\boldsymbol{K}^{\mathrm{AA}}$ in eq 16 did not become positive as numerical error accumulated, and $\left(\boldsymbol{K}^{\mathbf{A A}}\right)^{-1}$ had a large error, since $\left(\boldsymbol{K}^{\mathbf{A A}}\right)^{-1}$ was obtained by Cholesky's method for symmetric positive materices.

The calculated and experimental results for L5 agree well with each other as shown in Figure 6. In these temperature and frequency ranges, the error caused by limiting the range of integration in eq 14 after the past three periods of oscillation is not so large and the calculation can be carried out without much difficulty.

\section{Temperature-Dispersion Behavior of SBP Films}

The temperature-dispersion behavior of the SBP films was simulated and compared with experimental results of Arai et al. ${ }^{6}$ For the calculation the response function of PS homopolymers were used for polystyrene (S), polybutadiene (B), and poly(4vinylpyridine) $(\mathrm{P})$ domains by employing adequate $T_{\mathrm{g}}$ values for each. To distinguish the three phases we employed temperatures at which frozen microBrownian motions of the main chains begin to be librated as the $T_{\mathrm{g}}$ values in the WLF equation 19. The temperatures for $\mathrm{S}, \mathrm{B}, \mathrm{P}$ noted as $T_{\mathrm{g}}(\mathrm{S}), T_{\mathrm{g}}(\mathrm{B})$, and $T_{\mathrm{g}}(\mathrm{P})$ are 343,166 , and $393 \mathrm{~K}$, respectively. These values are somewhat lower than the commonly known $T_{\mathrm{g}}$ values of these polymers, which are the temperatures at which loss moduli $G^{\prime \prime}$ and/or $\tan \delta$ exhibit peaks in the temperature-dispersion curves.

As remarked in the previous section, calculation of the low-molecular-weight PS, L14, and L12, involved a large error so that the response function for L5 was used for P and that of L15 for the S and B phases in the calculations. In a film of "threelayer-lamellar" morphology each of the lamellae on the average run parallel to the film surface. For the calculation of the response to a sinusoidal shear strain, we assumed that the lamellae lie perpendicular to the plane of shear as shown in Figure 3. In an actual measurement, the complex tensile modulus $E^{*}$ was obtained instead of the complex shear modulus $G^{*}$. However, a small shear deformation can be decomposed into a tension and compression with the principal axis making a $45^{\circ}$ angle with the plane of the shear. This situation justifies our approximate model in which lamellae 


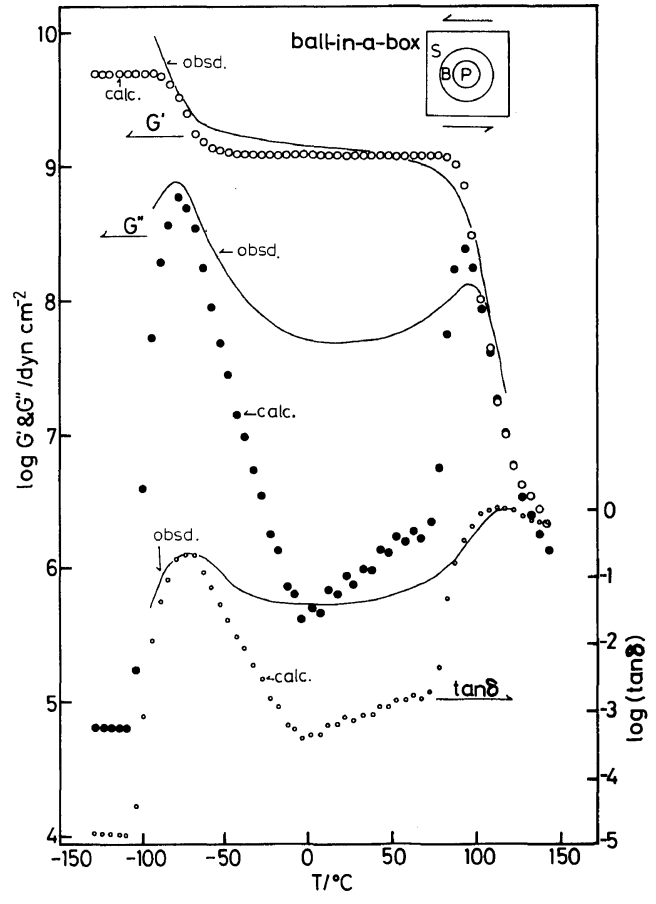

Figure 7. Comparison between the calculated and observed $^{6}$ temperature-dispersion behavior of an SBPspecimen of the "ball-in-a-box" morphology at $110 \mathrm{~Hz}$.

lie perpendicularly to the plane of shear. The contribution of higher harmonics to the calculated shear stress was very small, i.e., the ratio of the amplitudes to the fundamental harmonics was less than $10^{-4}$. Therefore we may say that the "total structure" exhibits a linear viscoelastic behavior under the sinusoidal shear strain examined here.

In Figures 7 and 8, the calculated and observed results of the temperature dispersion at $110 \mathrm{~Hz}$ of two films with the "ball-in-a-box" and "three-layerlamellar" morphologies are compared, respectively. The observed complex tensile moduli $E^{*}=E^{\prime}+i E^{\prime \prime}$ were reduced to complex shear moduli $G^{*}=G^{\prime}+i G^{\prime \prime}$ by simply assuming $G^{*}=E^{*} / 3$, i.e., the incompressibility and the linearity of the whole SBP system. The observed results are shown by solid curves, which exhibit the following features: The "ball-in-a-box" morphology shows only two transition peaks, presumably due to the glass transition of B and S phases, while the "three-layer-lamellar" morphology three transitions for all three phases. The calculated curves, especially that of $G^{\prime}$ simu-

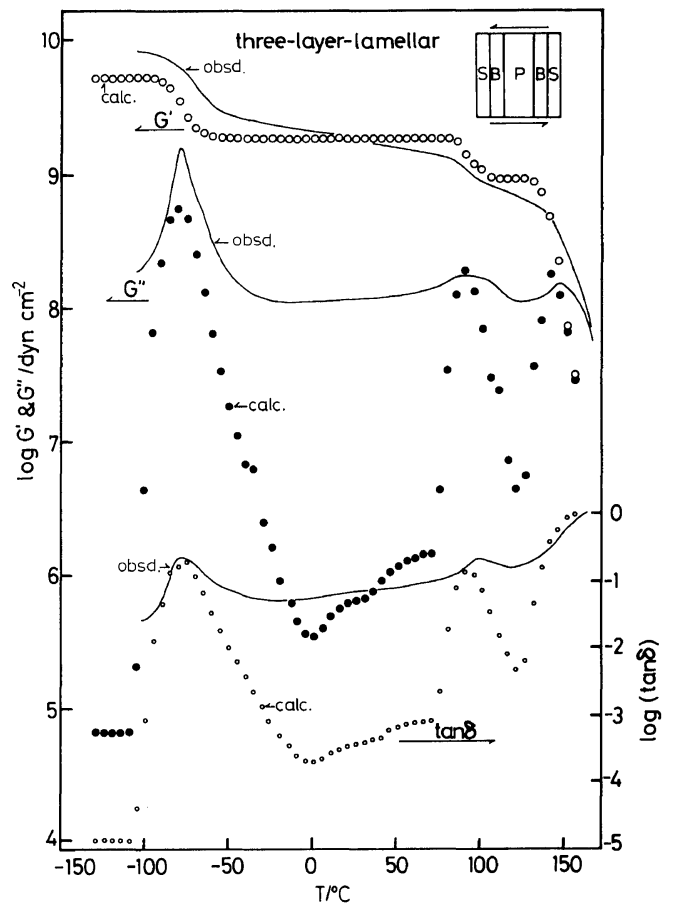

Figure 8. Comparison between claculated and observed temperature-dispersion behavior of an SBPspecimen of the "three-layer-lamellar" morphology at $110 \mathrm{~Hz}$.

lated these features very well. However, the drops of $G^{\prime \prime}$ and $\tan \delta$ between the transition peaks are very much larger in the calculated curves than in the observed ones. The calculation underestimates the mechanical loss considerably below the actual levels. This underestimation is presumably due to the neglect of part memories prior to the three periods in integrating eq 14 , and also to the assumption that $\mu^{\prime}$ is independent of $T$ below the $T_{\mathrm{g}}$.

Now returning to the problem of how only two transitions are activated in the "ball-in-a-box" morphology, while all three are activated in the "three-layer-lamellar" morphology. We examined the displacements of the domain boundaries of the models during one cycle of the sinusoidal shear deformation at four different temperatures. The results are shown in Figures 9 and 10 for the two morphologies. Below $T_{\mathrm{g}}(\mathrm{B})\left(-135^{\circ} \mathrm{C}\right)$ every phase deforms elastically and so do the whole models. As the temperature is raised above $T_{\mathrm{g}}(\mathrm{B})$, the difference becomes apparent: In the "ball-in-a-box" model, the P-sphere simply undergoes a rotatory oscil- 
(a) $-135^{\circ} \mathrm{C}<T_{\mathrm{g}}(\mathrm{B})<T_{\mathrm{g}}(\mathrm{S})<T_{\mathrm{g}}(\mathrm{P})$, period $=T$

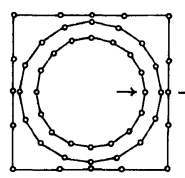

$t=0$

1

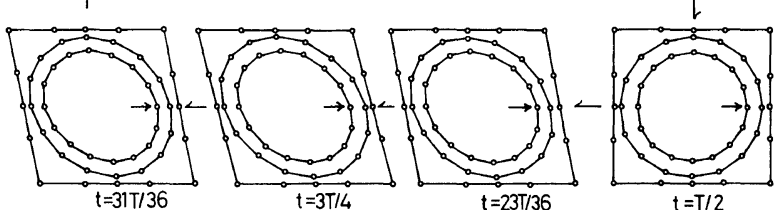

(b) $T_{\mathrm{g}}(\mathrm{B})<65^{\circ} \mathrm{C}<T_{\mathrm{g}}(\mathrm{S})<T_{\mathrm{g}}(\mathrm{P})$
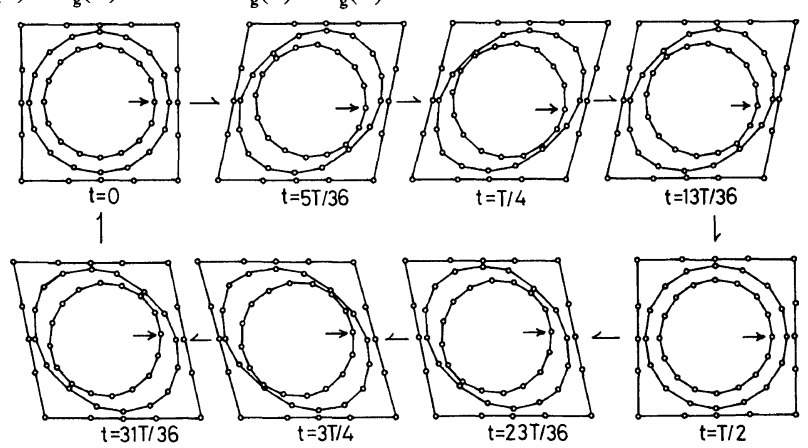

(c) $T_{\mathrm{g}}(\mathrm{B})<T_{\mathrm{g}}(\mathrm{S})<105^{\circ} \mathrm{C}<T_{\mathrm{g}}(\mathrm{P})$, period $=T$
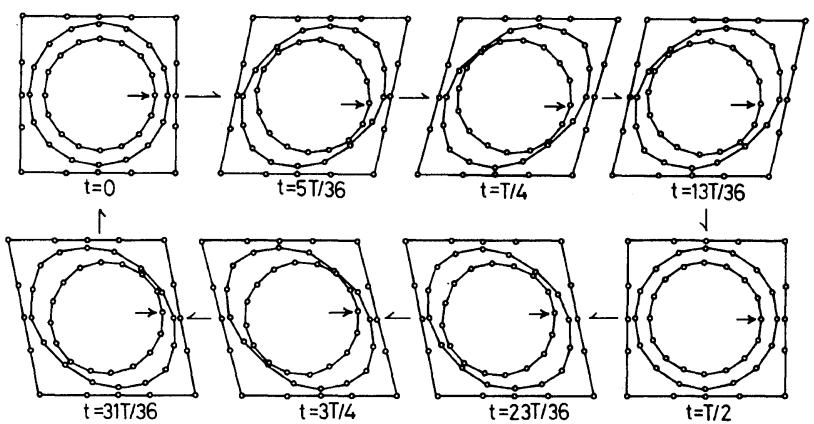

(d) $T_{\mathrm{g}}$ (B) $<T_{\mathrm{g}}$ (S) $<T_{\mathrm{g}}(\mathrm{P})<145^{\circ} \mathrm{C}$
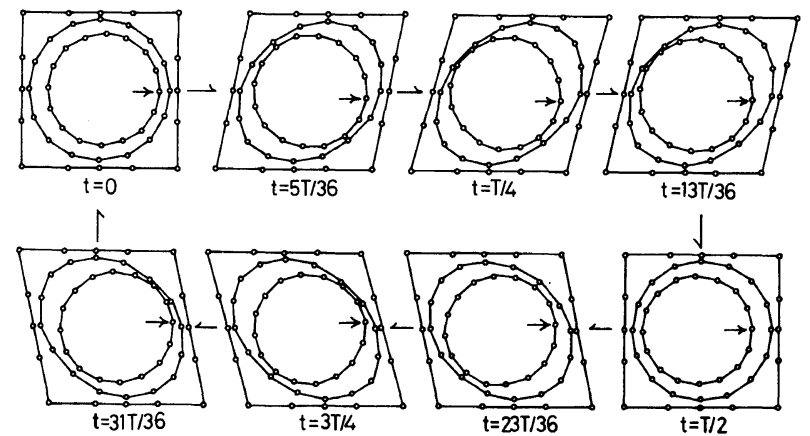

Figure 9. Schematic diagram, representing simulated deformation modes of phase-separated domains in the "ball-in-a-box" morphology at (a) -135 , (b) 65 , (c) 105 , and (d) $145^{\circ} \mathrm{C}$. The frequency of the sinusoidal deformation was $110 \mathrm{~Hz}$. 
Finite-Element Method Analysis of ABC Three-Block Polymer Systems

(a) $-135^{\circ} \mathrm{C}<T_{\mathrm{g}}(\mathrm{B})<T_{\mathrm{g}}(\mathrm{S})<T_{\mathrm{g}}(\mathrm{P})$, period $=T$

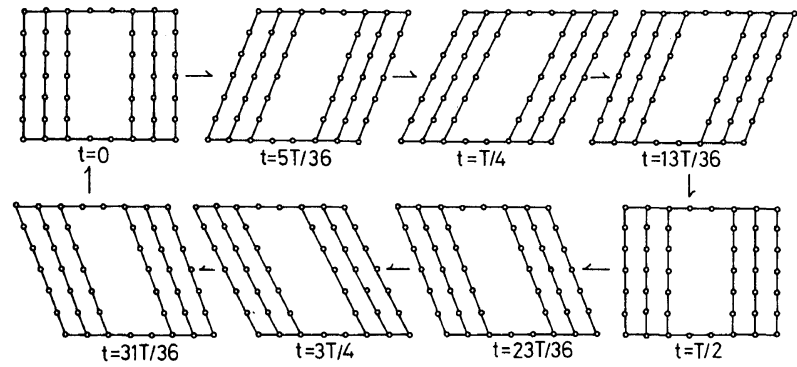

(b) $T_{\mathrm{g}}(\mathrm{B})<65^{\circ} \mathrm{C}<T_{\mathrm{g}}(\mathrm{S})<T_{\mathrm{g}}$ (P)

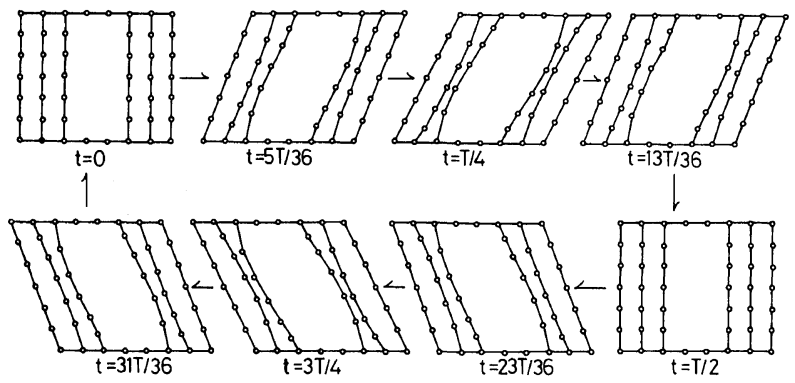

(c) $T_{\mathrm{g}}(\mathrm{B})<T_{\mathrm{g}}(\mathrm{S})<105^{\circ} \mathrm{C}<T_{\mathrm{g}}(\mathrm{P})$, period $=T$

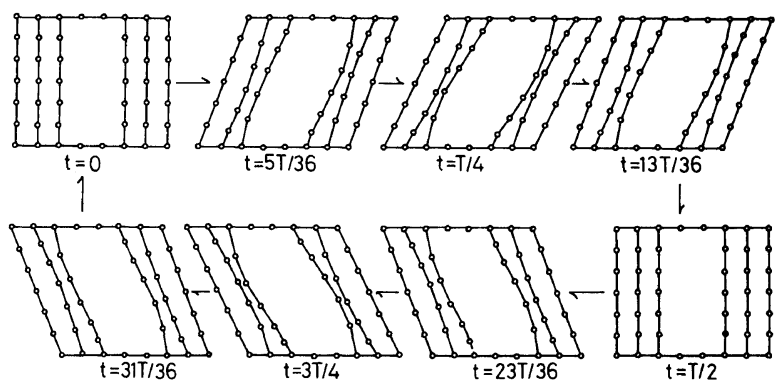

(d) $T_{\mathrm{g}}(\mathrm{B})<T_{\mathrm{g}}(\mathrm{S})<T_{\mathrm{g}}(\mathrm{P})<145^{\circ} \mathrm{C}$

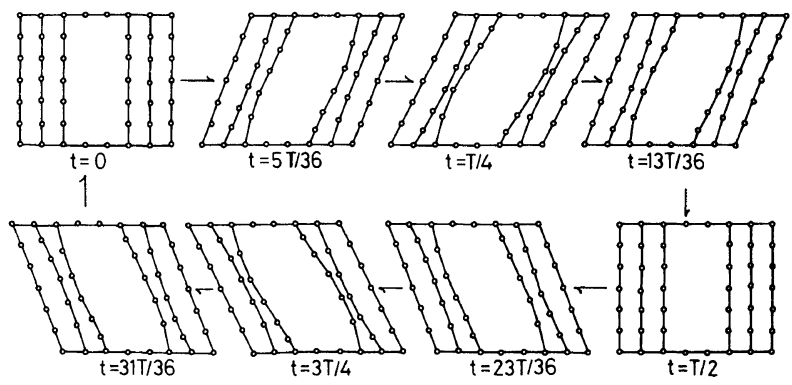

Figure 10. Schematic diagram representing simulated deformation modes of phase-separated domains in the "three-layer-lamella" morphology at (a) -135 , (b) 65 , (c) 105 , and (d) $145^{\circ} \mathrm{C}$. The frequency of the sinusoidal deformation was $110 \mathrm{~Hz}$. 
lational motion and swings back and forth in the softened B-sphere, thereby not contributing much to the dispersion. On the other hand, in the "threelayer-lamellar" model, the hard P lamella is still forced to deform even above $T_{\mathrm{g}}(\mathrm{S})$, and this contributes to the dispersion in the $T_{\mathrm{g}}(\mathrm{P})$ region. If the lamellae are laid parallel to the plane of shear, the hard P lamella simply swings back and forth and does not contribute to the dispersion. Thus the finite-element method is capable of predicting, at least semiquantitatively, the dynamic viscoelastic behavior of microphase-separated three-block polymer systems if the morphology and the mechanical properties of each component are understood.

\section{Nonlinearity of Phase-Separated Systems}

As remarked in the previous sections, phaseseparated SBP's under a small deformation show linear behavior. On the other hand, in decane or tetradecane solutions of a polystyrene-polybutadiene block copolymer (SB), S blocks are precipitated to form micelle structures. ${ }^{15,16}$ Such solutions usually show a nonlinear dynamic behavior even under a small-amplitude deformation: Lissajou's patterns are not elliptical and higher odd harmonics appear in the shear stress. This can be understood, at least semiquantitatively, as the behavior of a Bingham model connected in series with a Hookean spring. ${ }^{16}$ However, in view of the fact that the "three-phase structure" models do not show any nonlinearity in any temperature ranges as far as the small-amplitude deformation behavior is concerned, the multiphase structure itself is not responsible but a slippage of the micelles regularly arranged for the most part in a macrolattice structure appears to be responsible for such nonlinear behavior under the small-amplitude deformation.

Acknowledgement. We acknowledge with thanks the financial support from the Ministry of Education, Science, and Culture under the grants
B147087 (1976-1977) and B347081 (1978-1979) to TK. Numerical calculations were carried out at the Computation Center, Osaka University, using its ACOS series 77 NEAC system 900.

\section{REFERENCES}

1. O. C. Zienkiewics, "The Finite Element Method in Engineering Science," McGraw-Hill, Maidenhead, 1971.

2. C. S. Desai and T. F. Abul, "Introduction to the Finite Element Method," Van Nostrand Reinhold Co., Amsterdam, 1972.

3. J. J. Oden and T. Sato, Int. J. Solid Struct., 3, 479 (1976); P. B. Lindley, J. Strain Anal., 6, 45 (1977).

4. S. Kawabata, M. Matsuda, K. Tei, and H. Kawai, Polymer Preprint, Jpn., 27 (9), 1932 (1978); M. Matsuda and S. Kawabata, 28th Nihongomukyokai Koenyokoshu, Osaka, 1979, p 2.

5. K. Arai, T. Kotaka, Y. Kitano, and K. Yoshimura, Macromolecules, 13, 455 (1980).

6. K. Arai, T. Kotaka, Y. Kitano, and K. Yoshimura, Macromolecules, submitted.

7. See, for examples, A. S. Lodge, "Elastic Liquids," Academic Press, New York, 1964, Chapter 6; M. Yamamoto, "Buttai-no-Henkeigaku," Seibundoshinkosha, Tokyo, 1972, Chapter 6.

8. See, for example, S. Uemura and M. Takayanagi, $J$. Appl. Polym. Sci., 10, 113 (1966); R. A. Dickie, J. Appl. Polym. Sci., 17, 45 (1973); Y. T. Chen. J. Polym. Sci., Phys. Ed., 11, 2013 (1973).

9. See, also, M. Takayanagi and T. Kajiyama, "Mixing Law in Mechanical Properties," in "Composite Materials," Kagaku-Sosetsu, No. 8, NihonKagakukai, Tokyo, 1974.

10. D. A. G. Bruggemann, Ann. Phys., 29, 160 (1937).

11. E. H. Kerner, Proc. Phys. Soc., B69, 808 (1956).

12. S. Onogi, T. Masuda, and K. Kitamura, Macromolecules, 3 (2), 109 (1970).

13. S. Kakiuchi, T. Inoue, K. Kimura, T. Nishimura, S. Nomura, and T. Soen, 27th Reoroji-Toronkai Koenyoshishu, Yokohama, 1979, p 79.

14. J. D. Ferry, "Viscoelaștic Properties of Polymers," 2nd ed, John Wiley, New York, 1970.

15. T. Kotaka and J. L. White, Trans. Soc. Rheol., 17:4, 587-615 (1973).

16. H. Watanabe and T. Kotaka, Nihon Reoroji Gakkaishi, 8, 26 (1980). 